\title{
PERSONALISED DENTURES WITH BRANCHING TECHNIQUE
}

Puneet Chopra, Priyanka Chopra

1. Director, Department of Prosthodontics, Dental Care \& Implant Centre, Panchsheel Enclave, New Delhi.

2. Reader, Department of Periodontics, School of Dental Sciences. Sharda University, Greater Noida, Delhi.

\section{CORRESPONDING AUTHOR}

Dr. Puneet Chopra

C- 31, Panchsheel Enclave,

New Delhi- 110017.

E-mail: puneet.chopra@gmail.com

Ph: 00919810350090.

ABSTRACT: While the basic process of making dentures has changed little over the past several decades, new materials and techniques can help laboratories and clinicians provide functional, esthetic restorations that offer exceptional value to patients.

Unlike the conventional "linear" methods which follow specific steps in a cookbook fashion, the Branching Technique is a dynamic concept which can be adjusted to meet the clinical needs of each patient. This customized technique allows for a "trial" denture to be worn by each patient that is used to pinpoint and solve the patient's denture problems before the final denture is made. This individualized approach helps to eliminate disappointments or surprises. From the preliminary impressions and the "training" denture (it's actually a provisional denture) to the functional impressions and porcelain teeth to hold the patient's vertical dimension, the Branching Technique produces the most esthetically pleasing and functionally stable dentures possible.

KEY WORDS: Branching Technique, lingualized occlusion, retention, support, stability

INTRODUCTION: Complete denture prosthetics has been taught in schools the very same way since the turn of the century. ${ }^{1}$ The denture fabrication process is one that involves more appointments than most of the procedures. Normally there are six steps to accomplish so that the patient is satisfied with their new custom dentures.

While the basic process of making dentures has changed little over the past several decades, new materials and techniques can help laboratories and clinicians provide functional, esthetic restorations that offer exceptional value to patients. ${ }^{2}$ The success of complete dentures can be interpreted by the patient from two distinct view points: function and appearance.

One of the aims of prosthetic rehabilitation is to restore accurate phonation and dentofacial harmony. The incisors and canines take on a fundamental role in fulflling these functions. Everyday each person experiences the importance of these teeth in speaking and smiling (Martone, 1963; Pound, 1977b). Prosthetic dentistry has always tried to find a method capable of establishing the correct position of the anterior teeth in an edentulous arch. ${ }^{3}$

Adopting Frank Lloyd Wright's working philosophy that "form and function are one", Pound advanced the concept that function in the oral environment should not exist without aesthetic form, as is often seen in dentures. An alternative technique of denture fabrication is the Branching Technique which is a very individualized and personalized approach and was originally conceived by Dr. Earl Pound approximately 3 decades ago. The Branching Technique is a dynamic concept which can be adjusted to meet the clinical needs of each patient. 
PROSTHODONTIC PRINCIPLES: Three prominent prosthodontic principles are applied in the manufacture of dentures: stability, retention and support. Each principle is what helps keep dentures in the mouth, where they belong, allowing the patient to eat and speak normally again.

- Retention: Denture retention has been defined as 'resistance of a denture to vertical movement away from the tissues'4 and as 'that quality inherent in the prosthesis acting to resist the forces of dislodgement along the path of insertion's

- Stability, the second principle describes that quality of maintaining a constant character or position in the presence of forces that threaten to disturb it; the quality of being stable; to stand or endure. It is the quality of a removable dental prosthesis to be firm, steady, or constant, to resist displacement by functional horizontal or rotational stresses $^{6}$

- Support implies the resistance to displacement away from the basal tissue or underlying structures 6

THE THREE TECHNIQUES: Some clinicians have found satisfaction in fabricating complete dentures by mastering the classic "five-appointment method" taught to them in dental school and modified as needed or required for specific patients. ${ }^{2}$ A lot of denture wearers have difficulty in eating the foods that they want and need to eat, in speaking clearly, and with their appearance. To solve these problems, a more involved denture technique called the Branching Technique is used. The "branching technique," taught first by Pound and later by Dr. Walter "Jack" Turbyfill and others, allows for extended treatment and management of more complex patient problems. ${ }^{7}$ The accelerated techniques taught by Frush and Smudde allow for clinical efficiency while still managing difficult prosthodontic cases.

All three methods-the traditional five-appointment method, the branching technique and the accelerated technique-require attention to detail and the ability to efficiently fulfill patient expectations. ${ }^{2}$ Ultimate success in treatment appears to be attributable to a combination of excellent esthetics, fit and skill in patient management.

THE BRANCHING TECHNIQUE: The Branching Technique is a very individualized approach to denture fabrication originally conceived by Dr. Earl Pound approximately 30 years ago. In the modern practice of complete denture prosthetics, the edentulous patient is first provided with a provisional denture. -12 $^{-12}$ "training denture" is used to treat the inflamed gum tissue, establish the bite, develop the esthetics, and make an impression that perfectly fits the gums before the final denture is made. The "training denture" is a temporary denture that contains a soft, conforming material inside that is worn for several weeks while chewing food, smiling, talking etc. This provisional denture will allow the dentist to refine all of the functional aesthetic aspects of the denture to his or her and the patient's satisfaction. It basically provides a perfect blueprint for the final denture.This approach leads to patient happiness without the frustrating surprises of the past and makes the practice of denture prosthetics very predictable. While the patient wears the treatment denture, an added benefit is the creation of functional impressions .After final delivery of the denture, few, if any, post insertion adjustments are necessary.

This is not a quickie mass production technique, but designed for dentists who want the ultimate in full denture service for their patients.

HALLMARKS OF POUND TECHNIQUE: Hallmarks of the Pound technique include positioning the anterior teeth using the mandibular movements of speech, the use of lingualized occlusion, and the fabrication of diagnostic provisional dentures. ${ }^{2}$ 
The Branching Technique properly positions teeth in naturally contoured bases that fit and function well. Anterior teeth are arranged using the patient's speech patterns and esthetic needs. Posterior teeth are arranged for efficient function with minimal interferences.

The basis for determining the most ideal occlusal schemes for removable prosthetics borrows several of the accepted ideologies and procedures commonly used to stabilize and restore natural dentition. In partially edentulous cases, it is advisable to minimize any deleterious forces from the indirect retainers on the abutment teeth. ${ }^{13}$ With fully edentulous cases, the lingualized occlusion recommended by Turbyfill seems to provide the most successful results. ${ }^{14}$ Lingualized occlusion involves the use of more highly angled or 'sharper' denture teeth on the maxillary denture, against lower angled or more shallow anatomy on the mandibular denture. An anatomic maxillary posterior tooth (33-degree cuspid tooth) that occludes in a lingualized fashion into the central fossae of the lower is chosen.11,14 The esthetics is far superior to flat plane and other lesser degree teeth. The beautiful maxillary buccal cusps look natural. The purpose of the maxillary buccal cusp is esthetics, food manipulation, and overjet to prevent jaw biting. This produces a stable and functional occlusion with no interferences to rock the dentures. Porcelain posterior teeth resist wear and maintain vertical dimension of occlusion. This simulates a "mortar-pestle" action that aids chewing efficiency, yet can potentially minimize the lateral forces that can not only dislodge the dentures, but also create more sore spots.

One predictable impression technique that establishes uniform tissue support employs the various procedures developed by Pound and expounded by Turbyfill. ${ }^{15}$ The use of a soft liner in the treatment denture can produce dramatic improvement in the condition of the tissues that support the denture. Soft liners in the treatment dentures provide comfort and a secure fit, while reconditioning abused tissues. The healthy tissue will provide for a more comfortable and retentive denture-wearing experience.

Guidelines For Anterior Teeth Positioning

Proper contour and normal physiologic movement of the muscles enhances an esthetic denture, whereas improper support can detract from it severely. Positioning the anterior teeth using the mandibular movements of speech is a hallmark of the Pound technique, a method which has proven successful over decades. ${ }^{7,16}$

1. The pronunciation of the letter " $M$ " places the upper lip at rest at completion, illustrating the amount of display of the central incisors. Men should display $1 \mathrm{~mm}$ to $2 \mathrm{~mm}$; women $3 \mathrm{~mm}$ to $4 \mathrm{~mm}$.

2. The use of the letters " $F$ " and "V" illustrate the appropriate relationship of the incisal edges with the vermillion border. Ideally, the maxillary incisors brush lightly against the border during the formation of these letters.

3. The use of the letter "S" aids in the determination of appropriate vertical dimension through evaluation of the "speaking space." Both men and women should display $1 \mathrm{~mm}$ of clearance between the upper and lower incisors during the formation of this letter.

The mandibular anterior teeth are set using phonetics. Dawson ${ }^{17}$ noted that the vertical dimension of occlusion that has been lost can be regained by noting the closest speaking level and then establishing the vertical dimension of occlusion slightly more closed from that closest speaking position. Pound ${ }^{18}$ referred to this as the vertical dimension of speech, and since the teeth are not to touch while a person is speaking, then the vertical dimension of occlusion 
should be slightly more closed than the "S" position. The "S" position is the most intimate relationship of any teeth during speech. 19,20 $^{19}$

The vertical dimension of occlusion is very easy to determine since it is always less than the vertical dimension of speech. ${ }^{21-23}$ Therefore, when the anterior teeth are set to the "S" position, the mandible is retruded and closed down $2 \mathrm{~mm}$ to tooth contact or, if no teeth touch, merely closes in a centric relation $2 \mathrm{~mm}$ less than the vertical dimension of speech.

Two mandibular incisors are set to the "S" position. Pound defined the "S" position as the most intimate relationship of the teeth during speech. ${ }^{18}$ There are intimate relationships that occur between the incisal edges of the mandibular teeth and the incisal edges and lingual surfaces of the maxillary anterior teeth. This allows the dentist to verify the accuracy of the maxillary tooth arrangement and place the mandibular incisors in an anatomically natural position that produces an articulate speech pattern.

If a significant adjustment in the vertical dimension of occlusion (VDO) is required, the denture(s) can be adjusted to establish the appropriate VDO and incisal edge position on a trial basis. The clinician can articulate the denture before the impression procedure, equilibrate through occlusal reduction, or roughen the incisal and occlusal surfaces and add provisional material.

If adjustments in tooth position are desired in the new appliances, the clinician should record the desired changes with a study cast. Final dentures completed with porcelain teeth and custom tinted resin bases can restore one's appearance and confidence.

FUNCTIONAL IMPRESSION: A functional impression is created by applying a functional impression material to the fitting surface of an existing denture. This impression technique creates an impression under functional stresses. ${ }^{24}$ One functional impression material, HydroCast, was developed by Dr. Earl Pounds and Mr. Clark Smith in the 1950s, and remains one of the best kept secrets in dentistry. The described technique using this material can be used when the patient's existing dentures fit reasonably well. If any borders are overextended, they can be adjusted and polished. If any borders are short, they can be corrected by the addition of Triad.

1. Preparation of the Denture Base: Prepare the denture base by removing any extreme undercuts as would be performed for a reline impression. This preparation allows the denture to be removed from the master cast without damage to the cast or denture. Also, apply petroleum jelly to the teeth to facilitate easier removal of the tacky HydroCast material at completion.

2. Powder- Liquid Ratio: The material consists of a powder (fine-milled alginate) and liquid (ethanol) that are mixed at a ratio of 3:1. Mix the ingredients only enough to incorporate the components .

3. Manipulation of the Material: Allow the mixture to sit covered for a few minutes, until it reaches a manageable honey-like consistency. Then, load the material into the denture base. Border-mold the impression as normal during setting. The initial set takes 3 to 5 minutes.

4. Final Adjustment: On removal of the denture from the mouth, any premature pressure areas should be evident from the acrylic base bleed through. Adjust any premature contact areas with an acrylic carbide bur, mix and add new material, and reseat. Excess external material can be removed with a heated carving instrument.

5. Tissue Conditioning: After a satisfactory initial result, dismiss the patient with instructions to return in 4 to 5 days for evaluation of the functional result. The material 
does not reach a complete set until this period, thus the functional impression is accomplished. This technique also provides for tissue conditioning. Tissue conditioning occurs when a soft material applied to the fitting surface of a denture allows for more equal distribution of load and a return of the mucosal tissue to its normal position. ${ }^{24}$ Hydro-Cast is such a soft material. Tissue conditioning may need to be repeated over several visits to allow abused soft tissue to reach a satisfactory condition. Therefore, Steps 1 through 5 may need to be repeated over multiple office visits.

6. Bite Registration: On completion of the tissue conditioning and the final impression, take a bite registration, preferably with a vinyl or other static material as rubber base materials or bisacrylate formula based 25,26 . Then, pour the master casts and articulate the dentures.

7. In cases where upper and lower complete dentures are to be fabricated, additional steps are needed to ensure proper positioning of the maxillary anterior teeth on the new denture.

8. On articulation of the dentures, remove only the lower denture from the master cast.

9. 9.Adaptation of silicon putty: Place a roll of silicone lab putty or baseplate wax on the lower cast and close the articulator, capturing the upper denture teeth in the roll. In the case of a single maxillary denture, a silicone putty matrix can be accomplished by adapting the putty to the facial surfaces of the mandibular anterior teeth and the maxillary denture teeth.

10. Developing the study cast: Capture an alginate duplicate of the existing upper denture for the development of a study cast for the technician's use.

11. Anterior Teeth Positioning: The technician can place the incisal edges of the new anterior teeth in exactly the same position as the existing denture. If any adjustments in position are desired, the technician has a very specific starting point for reference and measurement.

The denture base is important in esthetics. ${ }^{27,28}$ In the 1950's the American Dr. Earl Pound innovated many techniques reproduction of living tissues in dentures is also made possible by the emergence of colour for creating natural looking dentures. After the boil-out and cleaning of the flask, one can "custom" stain the gingival areas. This is a relatively easy technique to learn but takes some time to master. The flask is the packed in the conventional manner and the staining acrylic becomes part of denture. The stains are called "Kayon". This method was developed by Dr. Pound in the 50's. There are 5 different stains

DISCUSSION: Patients that present for new dentures typically have old dentures with aberrant tooth positions, lost vertical dimension, poor esthetics, discomfort and, if their old dentures have plastic posterior teeth, there are heavy occlusal forces in the anterior due to the wear of the plastic teeth. Even worse, if the patient who presents for new dentures has no old dentures or teeth, there are no reference points to start from and denture fabrication can seem even more daunting for the practitioner.

The training denture allows the dentist and the patient the opportunity to evaluate all the factors mentioned above. Prior to the fabrication of the definitive dentures, any alterations deemed necessary from the "test drive" can be made. This ensures dentures of the highest quality for the patient.

In addition, when the training dentures are fabricated from the preliminary impressions, there is relief in the intaglio surface of the denture that provides room for a 
functional impression material (Hydrocast - Sultan Dental). Hydrocast is essentially a soft acrylic material that takes weeks to set and will record the differing redundancies of the various soft tissues in the mouth in function. It makes a very accurate final impression and border molds beautifully.

It has been argued that dentures with a lingualised reduced occlusal scheme may be advantageous as a lingualised occlusion is supposed to improve occlusal stability of complete dentures ${ }^{29}$. Another recently conducted study showed that dentures with lingualised occlusion scheme do appear to positively influence patients ratings of satisfaction concerning the stability of a set of complete dentures as compared to a traditionally bilaterally balanced occlusion. ${ }^{30}$

Certainly, the cost of fabricating a set of dentures in this manner will be greater, but there are many advantages of this technique. First, of course, is the quality of the final product for the patient. Second, the patient will have a back-up set of dentures that will fit beautifully if repairs are ever necessary to the definitive dentures.

When making tooth position changes to idealize esthetics and function, many times patients don't feel comfortable with such a dramatic change in their appearance. It sometimes takes a while for them to realize how much better they look with their lips supported or their vertical dimension opened back to where it had been previously or both. Fabricating a training denture allows the practitioner to get the patient most of the way to where he thinks the patient should be, and then additional changes can be made going from the training dentures to the definitive dentures.

Even the internal fit of the denture is functionally correct. It is made from an impression captured in action (during chewing, talking, smiling, swallowing, etc) rather than an impression that is pressed into the tissue for a few minutes while the patient is holding still. Predictability, comfort and satisfaction are finally possible. While the method of Branching Technique creates accurate final impressions and occlusal registrations, it does require a little more time and effort from the clinician and staff. ${ }^{16}$ This investment of time is returned when the patient returns for a predictable wax try-in, typically requiring little to no adjustment.

CONCLUSION: Given the use of a proven, functionally generated impression technique, extremely accurate and comfortable removable full and partial denture situations can be constructed with ease. For fully edentulous cases, the only additional requirement for success is providing a fully bilaterally balanced lingualized occlusal relationship. These are the tools that will help the treating dentist achieve predictable success, and provide the most comfortable and functional removable prosthetic alternative possible to an ever-expanding patient base.

\section{REFERENCES}

1. Boucher CO. Complete denture prosthodontics-the state of the art. J Prosthet Dent1975;34:372-83.

2. Melton AB. Current trends in removable prosthodontics. J Am Dent Assoc. 2000 Jun;131 Suppl:52S-56S.

3. F. Bassi, A. Rizzatti, G. Schierano \& G. Preti. Evaluation of the utility of cephalometric parameters in constructing complete denture. Part II: placement of anterior teeth Journal of Oral Rehabilitation 2001: 28; 349-353

4. Nairn R I, Shapiro N M S (eds). Prosthetic Dentistry Glossary. London: Quintessence, 1995.

5. Anon. Glossary of Prosthodontic Terms. 7th ed. J Prosthet Dent 1999; 81: 45-106. 
6. The Glossary of Prosthodontic Terms. J Prosthet Dent 2005; 94(1): 1-92

7. Turbyfill WF. Regaining pleasure and success for complete denture services. Int J Prosthodont 1989;2(5):474.

8. Appelbaum MB. The practical dynamics of the interim denture concept: a comparison with the conventional immediate denture technique. J Am Dent Assoc1983;106:826-30.

9. Hansen CA. Diagnostically restoring a reduced occlusal vertical dimension without permanently altering the existing dentures. J Prosthet Dent 1985;54: 671-3.

10. Pound E. Preparatory dentures: a protective philosophy. J Prosthet Dent 1965;15:5-18.

11. Pound E, Murrell GA. An introduction to denture simplification. J Prosthet Dent 1971;26:570-80.

12. Pound E, Murrell GA. An introduction to denture simplification. Phase II.

13. J ProsthetDent1973;29:598-607. Cecconi BT, Asgar K, Dootz E. The effect of partial denture clasp design on abutment tooth movement. J Prosthet Dent. 1971;25:44-56.

14. Clough HE, Knodle JM, Leeper SH, et al. A comparison of lingualized occlusion and monoplane occlusion in complete dentures. J Prosthet Dent 1983;50: 176-9.

15. Turbyfill WF. Impression techniques for removable partial dentures. Gen Dent. 2001;49:358-364.

16. David R. Avery. Using Existing Dentures for Wash Impression and Occlusal Registration Records .Inside Dentistry October 2008, Volume 4, Issue 9

17. Dawson P. Evaluation, diagnosis, and treatment of occlusal problems. St. Louis: CV Mosby, 1989

18. Pound E. Mandibular movements of speech and their seven related values. J Prosthet Dent 1966;16:835.

19. Pound E. Controlling anomalies of vertical dimension and speech. J Prosthet Dent 1976;36:124-35.

20. Pound E. Utilizing speech to simplify personalized denture service. J Prosthet Dent 1970;24:586-600.

21. 21) Murray CG. Re-establishing natural tooth position in the edentulous environment. Aust Dent J 1978;23: 415-21.

22. Murrell GA. Phonetics, function, and anterior occlusion. J Prosthet Dent 1974;32:23-31.

23. Sherman H. Phonetic capability as a function of vertical dimension in complete denture wearers-a preliminary report. J Prosthet Dent 1970;23:621-32.

24. Wilson HJ, Tomlin HR, Osborne J. Tissue conditioners and functional impression materials. Br Dent J. 1966;121:9-16.

25. Kois JC, Fan PP. Complete denture impressioning technique. Compend Contin Educ Dent. 1997;18(7):699-708

26. Massad J, Davis WJ, Lobel W, et al. Improving the stability of maxillary dentures: the use of polyvinyl siloxane impression materials for edentulous impressions. Dent Today. 2005;24(2):118-123.

27. Starcke EN Jr. The contours of polished surfaces of complete dentures: a review of the literature. J Am Dent Assoc 1970;81:155-60.

28. Zimmerman DE, Cotmore JM. Denture esthetics (I). Denture base contour. Quintessence Int 1982;13: 543-9.

29. Lang B.R. Complete Denture Occlusion. Dent Clin North Am 2004;48:641-665

30. Kimoto S, Gunji A, Yamakawa A, et al. Prospective clinical trial comparing lingualized occlusion to bilateral balanced occlusion in complete dentures: A pilot study. Int J Prosthodont 2006;19:103-109 